\title{
THE EXPONENTIAL STABILITY OF A COUPLED HYPERBOLIC/PARABOLIC SYSTEM ARISING IN STRUCTURAL ACOUSTICS
}

\author{
GEORGE AVALOS
}

\begin{abstract}
We show here the uniform stabilization of a coupled system of hyperbolic and parabolic PDE's which describes a particular fluid/structure interaction system. This system has the wave equation, which is satisfied on the interior of a bounded domain $\Omega$, coupled to a "parabolic-like" beam equation holding on $\partial \Omega$, and wherein the coupling is accomplished through velocity terms on the boundary. Our result is an analog of a recent result by Lasiecka and Triggiani which shows the exponential stability of the wave equation via Neumann feedback control, and like that work, depends upon a trace regularity estimate for solutions of hyperbolic equations.
\end{abstract}

\section{Introduction}

1.1. Statement of the Problem and Motivation. Let $\Omega$ be a bounded domain of $\mathbb{R}^{n}, n \geq 2$, with sufficiently smooth boundary $\Gamma=\Gamma_{0} \cup \Gamma_{1}$, with both $\Gamma_{i}$ open and nonempty. This paper is a continuation of our study, initiated in [1], of the following system consisting of a coupling between a wave and plate-like equation:

(1)

$$
\begin{cases}z_{t t}=\Delta z & \text { on }(0, \infty) \times \Omega \\ z(0, x)=z_{0}, z_{t}(0, x)=z_{1} & \text { on } \Omega \\ z(t, x)=0 & \text { on }(0, \infty) \times \Gamma_{0} \\ \frac{\partial z(t, x)}{\partial \nu}+\alpha z_{t}(t, x)=v_{t} & \text { on }(0, \infty) \times \Gamma_{1} \text { with } \alpha \geq 0 ;\end{cases}
$$

1991 Mathematics Subject Classification. Primary 93C20; Secondary 73K12, 73K50, 93C90.

Key words and phrases. Coupled hyperbolic/parabolic system, structural acoustics, exponential stability.

Received: April 6, 1996. 


$$
\begin{cases}v_{t t}=-\Delta^{2} v-\Delta^{2} v_{t}-z_{t} & \text { on }(0, \infty) \times \Gamma_{1} \\ v(0, x)=v_{0}, v_{t}(0, x)=v_{1} & \text { on } \Gamma_{1} \\ v(t, x)=\frac{\partial v(t, x)}{\partial \nu}=0 & \text { on }(0, \infty) \times \partial \Gamma_{1} .\end{cases}
$$

Note how this coupling above of two qualitatively different equations is accomplished by the velocity terms $z_{t}$ and $v_{t}$ on the active portion of the boundary $\Gamma_{1}$.

In [2], issues of well-posedness for (1)-(2) were settled, with initial data $\left[\overrightarrow{z_{0}}, \overrightarrow{v_{0}}\right] \equiv\left[z_{0}, z_{1}, v_{0}, v_{1}\right]$ determining the solution $[\vec{z}, \vec{v}] \equiv\left[z, z_{t}, v, v_{t}\right]$ to be in $H_{\Gamma_{0}}^{1}(\Omega) \times L^{2}(\Omega) \times H_{0}^{2}\left(\Gamma_{1}\right) \times L^{2}\left(\Gamma_{1}\right)$ (where $H_{\Gamma_{0}}^{1}(\Omega)=\left\{z \in H^{1}(\Omega)\right.$ э $z=0$ on $\left.\left.\Gamma_{0}\right\}\right)$. Here, we are concerned with the exponential decay of the solution $[\vec{z}, \vec{v}]$ to (1)-(2). Specifically, we wish to know: Defining the energy $E(\vec{z}, \vec{v}, t)$ of the system as

$$
\begin{aligned}
E(\vec{z}, \vec{v}, t)= & \int_{\Omega}\left[|\nabla z(t)|^{2}+\left|z_{t}(t)\right|^{2}\right] d \Omega \\
& +\int_{\Gamma_{1}}\left[|\Delta v(t)|^{2}+\left|v_{t}(t)\right|^{2}\right] d \Gamma_{1},
\end{aligned}
$$

do there exist positive constants $C$ and $\omega$ such that

$$
E(\vec{z}, \vec{v}, t) \leq C e^{-\omega t}\left\|\left[\begin{array}{l}
\overrightarrow{z_{0}} \\
\overrightarrow{v_{0}}
\end{array}\right]\right\|^{2} ?
$$

(where the norm above denotes that of the $H_{\Gamma_{0}}^{1}(\Omega) \times L^{2}(\Omega) \times H_{0}^{2}\left(\Gamma_{1}\right) \times$ $L^{2}\left(\Gamma_{1}\right)$-topology). This "structural acoustics" model is a variation of that derived by H.T. Banks et al (see [4], [5]) to mathematically describe the interaction between an acoustic field and its vibrating boundary, a physical phenonemon much studied nowadays within the realm of smart materials and structures and its accompanying numerical analysis. A simple PDE argument will reveal that the original system of Banks et al does not exhibit uniform decay, and hence the necessity for the supplanting with (1)-(2), focusing here on the case that the parameter $\alpha>0$.

A demonstration of exponential stability for the system (1)-(2) has important physical implications, as one would consequently be free to study the associated Linear Quadratic Regulator Problem (LQR) on Infinite Horizon. In the LQR for the structural acoustics model, boundary control is implemented via the placement of linear combinations of derivatives of delta functions in the beam equation of (2), so as to model the use of piezoelectric ceramic patches in inducing acoustic noise reduction (the LQR for finite time has been given a thorough treatment in [1]). Note here that the input operator which models the control action is "badly" unbounded, and the LQR is consequently not amenable to the recently developed treatments in [9]. The exponential stability of (1)-(2) is requisite in the analysis of the LQR for infinite time, which in turn could potentially yield a viable numerical approach (via a formulation of the appropriate Algebraic Riccati 
Equation) for obtaining approximations of the structural acoustics control problem. Moreover, the uniform stabilization of (1)-(2) is indispensable in future considerations of nonlinear versions of the model.

1.2. Preliminaries. In dealing with (1)-(2), we will consider throughout its equivalence with an abstract evolution equation, for the defining of which we will need the following background material:

- Let the operator $A: L^{2}(\Omega) \supset D(A) \rightarrow L^{2}(\Omega)$ be defined by

$$
A z=-\Delta z, D(A)=\left\{\left.z \in H^{2}(\Omega) \quad \ni z\right|_{\Gamma_{0}}=0,\left.\frac{\partial z}{\partial \nu}\right|_{\Gamma_{1}}=0\right\} .
$$

Note that $A$ is self-adjoint, positive definite, and hence the fractional powers of $A$ are well defined.

- By [10], we have the following characterization:

$$
D\left(A^{\frac{1}{2}}\right)=H_{\Gamma_{0}}^{1}(\Omega)=\left\{z \in H^{1}(\Omega) \text { э } z=0 \text { on } \Gamma_{0}\right\},
$$

(5) with $\|z\|_{D\left(A^{\frac{1}{2}}\right)}^{2}=\left\|A^{\frac{1}{2}} z\right\|_{L^{2}(\Omega)}^{2}=\int_{\Omega}|\nabla z|^{2} d \Omega=\|z\|_{H_{\Gamma_{0}}^{1}}^{2} \forall z \in D\left(A^{\frac{1}{2}}\right)$,

where the last equality in (5) follows from Poincaré's inequality.

- We define the map $N$ by

$$
z=N g \Longleftrightarrow \begin{cases}\Delta z=0 & \text { on } \Omega \\ \left.z\right|_{\Gamma_{0}}=0 & \text { on } \Gamma_{0} \\ \left.\frac{\partial z}{\partial \nu}\right|_{\Gamma_{1}}=g & \text { on } \Gamma_{1} ;\end{cases}
$$

elliptic theory will then yield that

$$
N \in \mathcal{L}\left(L^{2}\left(\Gamma_{1}\right), D\left(A^{\frac{3}{4}-\epsilon}\right)\right) \quad \forall \epsilon>0 .
$$

- Let $\gamma: H^{1}(\Omega) \rightarrow H^{\frac{1}{2}}\left(\Gamma_{1}\right)$ be the restriction to $\Gamma_{1}$ of the familiar Sobolev trace map; viz.

$$
\forall z \in H^{1}(\Omega), \gamma(z)= \begin{cases}\left.z\right|_{\Gamma_{1}} & \text { on } \Gamma_{1} \\ 0 & \text { on } \Gamma_{0}\end{cases}
$$

Then as is shown in [15], we have

$$
N^{*} A=\gamma(z) \forall z \in D\left(A^{\frac{1}{2}}\right) .
$$

- We set $\AA: L^{2}\left(\Gamma_{1}\right) \supset D(\AA) \rightarrow L^{2}\left(\Gamma_{1}\right)$ to be

$$
\AA=\Delta^{2}, D(\AA)=H^{4}\left(\Gamma_{1}\right) \cap H_{0}^{2}\left(\Gamma_{1}\right) ;
$$

$\AA$ is also self-adjoint, positive definite, and again by [10], we have the characterization

$$
\begin{aligned}
& D\left(\AA^{\frac{1}{2}}\right)=H_{0}^{2}\left(\Gamma_{1}\right), \\
& \text { with }\left\|\AA^{\frac{1}{2}} v\right\|_{L^{2}\left(\Gamma_{1}\right)}^{2}=\int_{\Gamma_{1}}|\Delta v|^{2} d \Omega=\|v\|_{H_{0}^{2}\left(\Gamma_{1}\right)}^{2} \forall v \in D\left(\AA^{\frac{1}{2}}\right) .
\end{aligned}
$$


- We define the energy spaces

$$
\begin{aligned}
& H_{1} \equiv D\left(A^{\frac{1}{2}}\right) \times L^{2}(\Omega) ; \\
& H_{0} \equiv D\left(\AA^{\frac{1}{2}}\right) \times L^{2}\left(\Gamma_{1}\right) .
\end{aligned}
$$

- We define $A_{1}: H_{1} \supseteq D\left(A_{1}\right) \rightarrow H_{1}$ and $A_{0}: H_{0} \supseteq D\left(A_{0}\right) \rightarrow H_{0}$ to be

$$
\begin{aligned}
& A_{1} \equiv\left[\begin{array}{cc}
0 & I \\
-A & -\alpha A N N^{*} A
\end{array}\right] \text { with } \\
& D\left(A_{1}\right)=\left\{\left[z_{1}, z_{2}\right]^{T} \in\left[D\left(A^{\frac{1}{2}}\right)\right]^{2} \ni z_{1}+\alpha N N^{*} A z_{2} \in D(A)\right\} ; \\
& A_{0} \equiv\left[\begin{array}{rr}
0 & I \\
-\AA & -\AA
\end{array}\right] \text { with } \\
& D\left(A_{0}\right)=\left\{\left[v_{1}, v_{2}\right]^{T} \in\left[D\left(\AA^{\frac{1}{2}}\right)\right]^{2} \ni v_{1}+v_{2} \in D(\AA)\right\} .
\end{aligned}
$$

With the above operator definitions, we set

$$
\begin{aligned}
& \mathcal{A}=\left[\begin{array}{cccc}
A_{1} & 0 & 0 \\
0 & 0 & & \gamma^{*} \\
0 & -\gamma & & A_{0}
\end{array}\right] \text { with } \\
& D(\mathcal{A})=\left\{\left[z_{1}, z_{2}, v, v_{2}\right]^{T} \in\left[D\left(A^{\frac{1}{2}}\right)\right]^{2} \times\left[D\left(\AA^{\frac{1}{2}}\right)\right]^{2}\right. \text { such that } \\
& \left.-z_{1}-\alpha N N^{*} A z_{2}+N v_{2} \in D(A) \text { and } v_{1}+v_{2} \in D(\AA)\right\} .
\end{aligned}
$$

If we take the initial data $\left[\overrightarrow{z_{0}}, \overrightarrow{v_{0}}\right]$ to be in $H_{1} \times H_{0}$ and $\vec{z}=\left[z, z_{t}\right], \vec{v}=$ $\left[v, v_{t}\right]$, we can use the definitions above to rewrite (1)-(2) abstractly as

$$
\begin{aligned}
& \frac{d}{d t}\left[\begin{array}{l}
\vec{z} \\
\vec{v}
\end{array}\right]=\mathcal{A}\left[\begin{array}{l}
\vec{z} \\
\vec{v}
\end{array}\right] \\
& {[\overrightarrow{z(0)}, \overrightarrow{v(0)}]=\left[\overrightarrow{z_{0}}, \overrightarrow{v_{0}}\right] .}
\end{aligned}
$$

Remark 1. The structure of $\mathcal{A}$ given in (19) clearly reflects the coupled nature of this particular system; The operator $A_{1}$ which models hyperbolic dynamics is linked via an unbounded coupling with the "elastic" operator $A_{0}$ which exhibits parabolic characteristics, this coupling being accomplished by "trace" operators.

From (19), the differential equations in (1)-(2) then have the following abstract representation:

$$
\begin{aligned}
& z_{t t}=-A z-\alpha A N N^{*} A z_{t}+A N v_{t} \text { on }(0, \infty) \times \Omega ; \\
& v_{t t}=-\AA \quad \AA-\AA v_{t}-N A^{*} z_{t} \text { on }(0, \infty) \times \Gamma_{1} .
\end{aligned}
$$

Regarding the well-posedness and strong stability of (1)-(2) and its equivalent form (19), we have the following recent result:

Theorem A. (see [2]) With $\alpha \geq 0$ in (1), 
(i) $\mathcal{A}$ given by (18) generates a $C_{0}$-semigroup of contractions $\left\{e^{\mathcal{A} t}\right\}_{t>0}$ on the energy space $H_{1} \times H_{0}$.

(ii) The semigroup $\left\{e^{\mathcal{A} t}\right\}_{t>0}$ is strongly stable; that is to say, $\forall\left[\overrightarrow{z_{0}}, \overrightarrow{v_{0}}\right] \equiv\left[z_{0}, z_{1}, v_{0}, v_{1}\right] \in H_{1} \times H_{0}$, one has

$$
\lim _{t \rightarrow \infty} e^{\mathcal{A} t}\left[\begin{array}{l}
\overrightarrow{z_{0}} \\
\overrightarrow{v_{0}}
\end{array}\right] \rightarrow 0 .
$$

1.3. Literature. The exponential stability for the individual components $A_{1}$ and $A_{0}$ have been well-established these past few years, but that of the entire structure $\mathcal{A}$ has not been addressed. Concerning the beam equation modelled by the "elastic" operator $A_{0}$, we have the result of $\mathrm{S}$. Chen and R. Triggiani in [8] that $A_{0}$ generates an analytic semigroup, which automatically provides for the exponential decay of the solution $\left[v, v_{t}\right]$ of the second-order system

$$
\begin{aligned}
& v_{t t}=-\Delta^{2} v-\Delta^{2} v_{t} \text { on }(0, \infty) \times \Gamma_{1} \\
& v(t, x)=\frac{\partial v(t, x)}{\partial v}=0 \text { on } \partial \Gamma_{1} \\
& {\left[v(0), v_{t}(0)\right]=\left[v_{0}, v_{1}\right] \in H_{1} \times H_{0} .}
\end{aligned}
$$

For the wave equation with $L^{2}\left(0, T ; L^{2}(\Omega)\right)$-Neumann feedback control; viz.

$$
\begin{array}{ll}
z_{t t}=\Delta z & \text { on }(0, \infty) \times \Omega \\
z(0, x)=z_{0}, z_{t}(0, x)=z_{1} & \text { on } \Omega \\
z(t, x)=0 & \text { on }(0, \infty) \times \Gamma_{0} \\
\frac{\partial z(t, x)}{\partial \nu}=-\alpha z_{t}(t, x) & \text { on }(0, \infty) \times \Gamma_{1} \alpha>0 ;
\end{array}
$$

G. Chen in [7] proved the exponential stability of solutions (24) under the geometrical conditions that $\Omega$ be "star-shaped". J. Lagnese in [11], and subsequently, R. Triggiani in [15] through an alternate proof, showed the uniform stabilization of (24) under the lessened constraint that there exist a $\left[C^{2}(\bar{\Omega})\right]^{n}$-vector field $h(x)$ such that

(j) $h \cdot \nu \leq 0$ on $\Gamma_{0}$ where $\nu$ denotes the unit-normal vector to $\Gamma$;

(jj) $h$ is parallel to $\nu$ on $\Gamma_{1}$;

(jjj) The Jacobian matrix $H(x)$ of $h(x)$ is uniformly positive definite on $\bar{\Omega}$.

Also, C. Bardos, G. Lebeau and J. Rauch in [6] have derived stability results for wave equations with more general boundary conditions than those in (24), under the assumptions of geometric optics; however the techniques used in the proofs therein are not easily adaptable to our particular situation, based as they are on microlocal analysis and the propagation of singularities. Instead, we shall use the approach of I. Lasiecka and R. Triggiani in [12], who 
have shown the exponential decay of solutions of (24) without the constraint (jj). This result is proved by using the standard multipliers $h \cdot \nabla z$ and $z$ div $h$, and invoking a deep (pseudodifferential) trace estimate which we state here for future reference:

Lemma A. (see [12]) Let $\epsilon>0$ be arbitrarily small. Let $z$ solve an arbitrary second-order hyperbolic equation on $(0, T)$ with smooth space-dependent coefficients. Then with $Q_{T} \equiv(0, T) \times \Omega$,

$$
\begin{gathered}
\int_{\epsilon}^{T-\epsilon} \int_{\Gamma_{1}}\left(\frac{\partial z}{\partial \tau}\right)^{2} d \Gamma_{1} d t<C_{T, \epsilon}\left\{\int_{0}^{T} \int_{\Gamma_{1}}\left\{\left(\frac{\partial z}{\partial \nu}\right)^{2}+z_{t}^{2}\right\} d \Gamma_{1} d t\right. \\
\left.+\|z\|_{H^{\frac{1}{2}+\epsilon}\left(Q_{T}\right)}^{2}\right\},
\end{gathered}
$$

where the $\epsilon$ on the left of (25) need not be the same as the $\epsilon$ for the $Q_{T}$-norm on the right, and where $\frac{\partial}{\partial \tau}$ denotes the tangential, and $\frac{\partial}{\partial \nu}$ the co-normal derivative.

It is this control of the tangential derivative provided above that allows one to forego the condition (jj) and generate the desired bound on the energy. In what follows, we will use critically the fact that Lemma A is applicable to the coupled wave equation given in (1).

\subsection{Statement of Main Result.}

Theorem 1. With $\alpha>0$ in (1), suppose there exists a vector field $h=$ $\left[h_{1}(x), h_{2}(x), \ldots, h_{n}(x)\right] \in\left[C^{2}(\bar{\Omega})\right]^{n}$ satisfying $(j)$ and (jij) only. Then, the semigroup $\left\{e^{\mathcal{A} t}\right\}_{t>0}$ generated by the operator $\mathcal{A}$ (defined in (18)) is exponentially stable; that is to say, there exists positive constants $C$ and $\omega$ such that the solution $[\vec{z}, \vec{v}]$ of (1)-(2) satisfies

$$
E(\vec{z}, \vec{v}, t)=\left\|e^{\mathcal{A} t}\left[\begin{array}{c}
\vec{z} \\
\vec{v}
\end{array}\right]\right\|_{H_{1} \times H_{0}}^{2} \leq C e^{-\omega t}\left\|\frac{\overrightarrow{z_{0}}}{\overrightarrow{v_{0}}}\right\|_{H_{1} \times H_{0}}^{2} .
$$

Note that the proof of Theorem 1 is independent of the strong stability result posted in Lemma $\mathrm{A}$, wherein there is no imposition of geometrical conditions. As will be explained below, to demonstrate the exponential stability, it will suffice to show that there exists a $T, 0<T<\infty$ and corresponding constant $C_{T}$ such that

$$
E(\vec{z}, \vec{v}, T) \leq C_{T} \int_{0}^{T}\left[\left\|z_{t}\right\|_{L^{2}\left(\Gamma_{1}\right)}^{2}+\left\|\AA^{\frac{1}{2}} v_{t}\right\|_{L^{2}\left(\Gamma_{1}\right)}^{2}\right] d t .
$$

To obtain (27), we will rely on the strong damping provided by the beam equation in (2) combined with a multiplier method for the wave equation in (1) to extract a preliminary upper bound on the energy $E(\vec{z}, \vec{v}, T)$. This upper bound, besides containing the RHS of (27), also includes the tangential derivative of $z$ and lower order terms. We then use Lemma A, in a very similar way to that done in [12], to estimate $\frac{\partial z}{\partial \tau}$ in terms of the RHS 
of (27) and more lower order terms, and finally eliminate these lower order terms through a compactness/uniqueness argument.

Remark 2. The assumptions (j) and (jjj) on the vector field $h$ will be satisfied if $\Gamma_{0}$ is a sufficiently small portion of $\Gamma$, viz. if meas $\left(\Gamma_{0}\right) \leq \frac{1}{2}$ meas $(\Gamma)$.

Remark 3. In estimating the energy contribution of (1), one could also proceed as in [11] and [15] to eventually arrive at the uniform stabilization of (1)-(2) under all the geometrical conditions $(\mathrm{j})-(\mathrm{jjj})$. To reiterate, it is the abstract trace estimate (25) which helps to yield the stronger result by eliminating outright the condition (jj).

In proving Theorem 1, we will, without loss of generality, take $\alpha \equiv 1$, as one will see in the proofs below that the value of $\alpha$ is irrelevant, so long as it is positive.

\section{Proof of Main Result}

Throughout, the initial data $\left[\overrightarrow{z_{0}}, \overrightarrow{v_{0}}\right]$ is taken to be in $D(\mathcal{A})$, which provides that $\left[z, z_{t}, v, v_{t}\right] \in C([0, T] ; D(\mathcal{A}))$, and $\left[z_{t}, z_{t t}, v_{t}, v_{t t}\right] \in C\left([0, T] ; H_{1} \times\right.$ $\left.H_{0}\right)$. Proving the results below and subsequently Theorem 1 for this special case will be conclusive, as we can then extend the obtained results by density to hold for all initial data in $H_{1} \times H_{0}$.

We first establish a preliminary concerning the a priori regularity of the velocity terms $z_{t}$ and $v_{t}$ which will be used frequently in the work ahead.

Proposition 1. With $[\vec{z}, \vec{v}]$ the solution of (1)-(2) (guaranteed by Theorem A.(i)), we have

The $\operatorname{map}\left\{\overrightarrow{z_{0}}, \overrightarrow{v_{0}}\right\} \rightarrow\left\{\left.z_{t}\right|_{\Gamma_{1}}, v_{t}\right\} \in$

$\mathcal{L}\left(H_{1} \times H_{0}, L^{2}\left(0, \infty ; L^{2}\left(\Gamma_{1}\right) \times D\left(\AA^{\frac{1}{2}}\right)\right)\right)$. Indeed, we have $\forall 0<T<\infty$,

$$
\begin{array}{r}
2 \int_{0}^{T}\left[\left\|\left.z_{t}\right|_{\Gamma_{1}}\right\|_{L^{2}\left(\Gamma_{1}\right)}^{2}+\left\|\AA^{\frac{1}{2}} v_{t}\right\|_{L^{2}\left(\Gamma_{1}\right)}^{2}\right] d t \\
=E(\vec{z}, \vec{v}, 0)-E(\vec{z}, \vec{v}, T) .
\end{array}
$$

Proof: We have by multiplying $(20)$ by $z_{t},(21)$ by $v_{t}$, and integrating from 0 to $T$ :

$$
\begin{aligned}
& \begin{aligned}
(29) \int_{0}^{T} & \left\|N^{*} A z_{t}\right\|_{L^{2}\left(\Gamma_{1}\right)}^{2} d t=\int_{0}^{T}\left\langle A N v_{t}, z_{t}\right\rangle\left[D\left(A^{\frac{1}{2}}\right)\right]^{\prime} \times D\left(A^{\frac{1}{2}}\right) \\
& +\frac{1}{2}\left[\left\|A^{\frac{1}{2}} z_{0}\right\|_{L^{2}(\Omega)}^{2}+\left\|z_{1}\right\|_{L^{2}(\Omega)}^{2}-\left\|A^{\frac{1}{2}} z(T)\right\|_{L^{2}(\Omega)}^{2}-\left\|z_{t}(T)\right\|_{L^{2}(\Omega)}^{2}\right] \\
(30) \int_{0}^{T}\left\|\AA^{\frac{1}{2}} v_{t}\right\|_{L^{2}\left(\Gamma_{1}\right)}^{2}=-\int_{0}^{T}\left(N^{*} A z_{t}, v_{t}\right) & L_{L^{2}\left(\Gamma_{1}\right)} d t \\
& +\frac{1}{2}\left[\left\|\AA^{\frac{1}{2}} v_{0}\right\|_{L^{2}\left(\Gamma_{1}\right)}^{2}+\left\|v_{1}\right\|_{L^{2}\left(\Gamma_{1}\right)}^{2}-\left\|\AA^{\frac{1}{2}} v(T)\right\|_{L^{2}\left(\Gamma_{1}\right)}^{2}-\left\|v_{t}(T)\right\|_{L^{2}\left(\Gamma_{1}\right)}^{2}\right] .
\end{aligned}
\end{aligned}
$$


Considering the definition of $E$ given in (3) and the characterizations (5), (11) and (9), the desired relation (28) is obtained after the addition of the quantities in (29)-(30). The asserted continuity of the map $\left\{\overrightarrow{z_{0}}, \overrightarrow{v_{0}}\right\} \rightarrow$ $\left\{\left.z_{t}\right|_{\Gamma_{1}}, v_{t}\right\}$ is consequently deduced from (28) and the contraction of the semigroup $\left\{e^{\mathcal{A} t}\right\}_{t \geq 0}$.

2.1. Proof of Theorem 1. A standard argument has that to prove the exponential decay rate in (26), it will suffice to show that exists a time $0<T<\infty$ and a corresponding positive constant $C_{T}$ such that for all initial data in $H_{1} \times H_{0}$,

$$
E(\vec{z}, \vec{v}, T) \leq \eta E(\vec{z}, \vec{v}, 0) \text { with } \eta<1
$$

given Proposition 1, it will in turn suffice to show that there exists a time $0<T<\infty$ and a corresponding positive constant $C_{T}$ such that

$$
E(\vec{z}, \vec{v}, T) \leq C_{T} \int_{0}^{T}\left[\left\|\left.z_{t}\right|_{\Gamma_{1}}\right\|_{L^{2}\left(\Gamma_{1}\right)}^{2}+\left\|\AA^{\frac{1}{2}} v_{t}\right\|_{L^{2}\left(\Gamma_{1}\right)}^{2}\right] d t
$$

to which end we proceed to work.

Throughout, we will make use of the denotations $Q_{T} \equiv(0, T) \times \Omega, \Sigma_{T} \equiv$ $(0, T) \times \Gamma$ and $\Sigma_{i T} \equiv(0, T) \times \Gamma_{i}, i=0,1$.

Lemma 1. There exists a positive constant $C$, independent of time, such that $\forall 0<T<\infty$

$$
\begin{aligned}
\int_{0}^{T}[ & \left.\left\|\AA^{\frac{1}{2}} v\right\|_{L^{2}\left(\Gamma_{1}\right)}^{2}+\left\|v_{t}\right\|_{L^{2}\left(\Gamma_{1}\right)}^{2}\right] d t \leq C[E(\vec{z}, \vec{v}, T) \\
& +E(\vec{z}, \vec{v}, 0)+\int_{0}^{T}\left(\left\|\left.z_{t}\right|_{\Gamma_{1}}\right\|_{L^{2}\left(\Gamma_{1}\right)}^{2}+\left\|\AA^{\frac{1}{2}} v_{t}\right\|_{L^{2}\left(\Gamma_{1}\right)}^{2}\right) d t \\
& \left.+\|v\|_{L^{2}\left(\Sigma_{1 T}\right)}^{2}\right] .
\end{aligned}
$$

Proof. Trivially, we have that

$$
\int_{0}^{T}\left\|v_{t}\right\|_{L^{2}\left(\Gamma_{1}\right)}^{2} d t \leq\left\|\AA^{-\frac{1}{2}}\right\| \int_{0}^{T}\left\|\AA^{\frac{1}{2}} v_{t}\right\|_{L^{2}\left(\Gamma_{1}\right)}^{2} d t .
$$

Moreover, multiplying (21) by $v$ and integrating from 0 to $T$ yields

$$
\begin{gathered}
\int_{0}^{T}\left\|\AA^{\frac{1}{2}} v\right\|_{L^{2}\left(\Gamma_{1}\right)}^{2} d t=\int_{0}^{T}\left\|v_{t}\right\|_{L^{2}\left(\Gamma_{1}\right)}^{2} d t-\left[\left(v_{t}, v\right)_{L^{2}\left(\Gamma_{1}\right)}\right]_{0}^{T} \\
+\frac{1}{2}\left[\left\|\AA^{\frac{1}{2}} v_{t}\right\|_{L^{2}\left(\Gamma_{1}\right)}^{2}\right]_{0}^{T}-\int_{0}^{T}\left(N^{*} A z_{t}, v\right)_{L^{2}\left(\Gamma_{1}\right)} d t
\end{gathered}
$$


using (34), Poincare's inequality, the definition of the energy $E$ in (3), and Cauchy-Schwarz on the RHS of (35) thus yields

$$
\begin{aligned}
\int_{0}^{T}\left\|\AA^{\frac{1}{2}} v\right\|_{L^{2}\left(\Gamma_{1}\right)}^{2} d t \\
\leq C\left[\int_{0}^{T}\left(\left\|N^{*} A z_{t}\right\|_{L^{2}\left(\Gamma_{1}\right)}^{2}+\left\|\AA^{\frac{1}{2}} v_{t}\right\|_{L^{2}\left(\Gamma_{1}\right)}^{2} d t\right)\right. \\
\left.\quad+E(\vec{z}, \vec{v}, T)+E(\vec{z}, \vec{v}, 0)+\|v\|_{L^{2}\left(\Sigma_{1 \mathrm{~T}}\right)}^{2}\right]
\end{aligned}
$$

where $C$ is independent of time. The result follows upon coupling (34) and (36), and further recalling the characterization (9).

Lemma 2. There exists a constant $C$, independent of time, such that

$$
\begin{aligned}
\int_{Q_{T}} z_{t}^{2} d Q_{T} \leq & C\left[\int_{0}^{T}\left(\left\|\left.z_{t}\right|_{\Gamma_{1}}\right\|_{L^{2}\left(\Gamma_{1}\right)}^{2}+\left\|\AA^{\frac{1}{2}} v_{t}\right\|_{L^{2}\left(\Gamma_{1}\right)}^{2}\right) d t\right. \\
& +\int_{\Sigma_{1 T}}\left(\frac{\partial z}{\partial \tau}\right)^{2} d \Sigma_{1 T}+E(\vec{z}, \vec{v}, T) \\
& \left.+E(\vec{z}, \vec{v}, 0)+\|z\|_{L^{2}\left(Q_{T}\right)}^{2}\right] .
\end{aligned}
$$

Proof. With the given vector field $h(x)$ satisfying $(\mathrm{j})$ and (jjj), we have upon multiplying the wave equation in (1) by $h \cdot \nabla z$ the standard identity (see [15], Appendix A):

$$
\begin{aligned}
& \int_{Q_{T}} H \nabla z \cdot \nabla z d Q_{T}=\int_{\Sigma_{T}} \frac{\partial z}{\partial \nu} h \cdot \nabla z d \Sigma_{T} \\
& +\frac{1}{2} \int_{\Sigma_{T}} z_{t}^{2} h \cdot \nu d \Sigma_{T}-\frac{1}{2} \int_{\Sigma_{T}}|\nabla z|^{2} h \cdot \nu d \Sigma_{T} \\
& -\frac{1}{2} \int_{Q_{T}}\left\{z_{t}^{2}-|\nabla z|^{2}\right\} \operatorname{div} h d Q_{T}-\left[\left(z_{t}, h \cdot \nabla z\right)_{L^{2}(\Omega)}\right]_{0}^{T} .
\end{aligned}
$$

As $\left[z, z_{t}\right] \in D\left(A^{\frac{1}{2}}\right) \times D\left(A^{\frac{1}{2}}\right)$, we then note that

$$
\text { on } \Sigma_{0 T}: z_{t}=0 ; \quad\left|\frac{\partial z}{\partial \nu}\right|=|\nabla z| ; h \cdot \nabla z=h \cdot \nu \frac{\partial z}{\partial \nu} ;
$$

and thus

$$
\begin{aligned}
\int_{\Sigma_{0 T}} \frac{\partial z}{\partial \nu} h \cdot \nabla z d \Sigma_{0 T} & +\frac{1}{2} \int_{\Sigma_{0 T}} z_{t}^{2} h \cdot \nu d \Sigma_{0 T} \\
& -\frac{1}{2} \int_{\Sigma_{0 T}}|\nabla z|^{2} h \cdot \nu d \Sigma_{0 T} \\
= & \frac{1}{2} \int_{\Sigma_{0 T}}|\nabla z|^{2} h \cdot \nu d \Sigma_{0 T} \leq 0
\end{aligned}
$$


after using the condition (j). Inserting the inequality (39) into (38) will therefore yield

$$
\begin{aligned}
\int_{Q_{T}} H & \nabla z \cdot \nabla z d Q_{T} \leq \int_{\Sigma_{1 T}} \frac{\partial z}{\partial \nu} h \cdot \nabla z d \Sigma_{1 T}+\frac{1}{2} \int_{\Sigma_{1 T}} z_{t}^{2} h \cdot \nu d \Sigma_{1 T} \\
& -\frac{1}{2} \int_{\Sigma 1_{T}}|\nabla z|^{2} h \cdot \nu d \Sigma_{1 T}-\frac{1}{2} \int_{Q_{T}}\left\{z_{t}^{2}-|\nabla z|^{2}\right\} \operatorname{div} h d Q_{T} \\
& -\left[\left(z_{t}, h \cdot \nabla z\right)_{L^{2}(\Omega)}\right]_{0}^{T} ;
\end{aligned}
$$

hence, using the condition (jjj), the Neumann B.C. in (1), the definition of $E$ and Cauchy-Schwarz gives us, after estimating both sides of (40),

$$
\begin{aligned}
& \rho \int_{Q_{T}}|\nabla z|^{2} d Q_{T} \leq C\left[\int_{\Sigma_{1 T}}\left[z_{t}^{2}+v_{t}^{2}\right] d \Sigma_{1 T}+\int_{\Sigma_{1 T}}|\nabla z|^{2} d \Sigma_{1 T}\right. \\
& \left.+E(\vec{z}, \vec{v}, T)+E(\vec{z}, \vec{v}, 0)-\frac{1}{2} \int_{Q_{T}}\left\{z_{t}^{2}-|\nabla z|^{2}\right\} \operatorname{div} h d Q_{T}\right] .
\end{aligned}
$$

Now, to handle the last term on the RHS of (41), we can multiply the wave equation (1) by $z \operatorname{div} \tilde{h}$, where $\tilde{h} \in\left[C^{2}(\bar{\Omega})\right]^{n}$ is arbitrary, and integrate by parts to obtain

$$
\begin{aligned}
& \int_{Q_{T}}\left\{z_{t}^{2}-|\nabla z|^{2}\right\} \operatorname{div} \tilde{h} d Q_{T}=\left[\left(z_{t}, z \operatorname{div} \tilde{h}\right)_{L^{2}(\Omega)}\right]_{0}^{T} \\
& +\int_{Q_{T}} z \nabla(\operatorname{div} \tilde{h}) \cdot \nabla z d Q_{T}-\int_{\Sigma_{1 T}} \frac{\partial z}{\partial \nu} z \operatorname{div} \tilde{h} d \Sigma_{1 T}
\end{aligned}
$$

after using Green's Theorem and the identity $\nabla(z \operatorname{div} \tilde{h}) \cdot \nabla z=z \nabla(\operatorname{div} \tilde{h})$. $\nabla z+|\nabla z|^{2} \operatorname{div} \tilde{h}$. We thus have upon majorizing the RHS of (42) with the use of Poincaré's inequality and the Neumann B.C. in (1),

$$
\begin{aligned}
& \left|\int_{Q_{T}}\left\{z_{t}^{2}-|\nabla z|^{2}\right\} \operatorname{div} \tilde{h} d Q_{T}\right| \\
& \leq C_{1}\left[\int_{Q_{T}} z^{2} d Q_{T}+\int_{\Sigma_{1 T}}\left[z_{t}^{2}+v_{t}^{2}\right] d \Sigma_{1 T}\right. \\
& \quad+E(\vec{z}, \vec{v}, T)+E(\vec{z}, \vec{v}, 0)]+2 \epsilon \int_{Q_{T}}|\nabla z|^{2} d Q_{T},
\end{aligned}
$$

where $\epsilon>0$ is arbitrarily small, and where the noncrucial dependence of $C_{1}$ upon $\epsilon$ has not been noted. Thus for $\epsilon$ small enough, adding the inequalities 
(41) and (43) together (with $\tilde{h} \equiv h$ ) yields

$$
\begin{aligned}
& (\rho-2 \epsilon) \int_{Q_{T}}|\nabla z|^{2} d Q_{T} \\
& \leq C_{1}\left[\int_{\Sigma_{1 T}}\left[z_{t}^{2}+v_{t}^{2}\right] d \Sigma_{1 T}+\int_{\Sigma_{1 T}}|\nabla z|^{2} d \Sigma_{1 T}\right. \\
& \left.\quad+E(\vec{z}, \vec{v}, T)+E(\vec{z}, \vec{v}, 0)+\int_{Q_{T}} z^{2} d Q_{T}\right] .
\end{aligned}
$$

Moreover, (43) (where $\tilde{h}$ is such that $\operatorname{div} \tilde{h}=1$ ) and (44) together gives

$$
\begin{aligned}
& (\rho-2 \epsilon) \int_{Q_{T}} z_{t}^{2} d Q_{T} \\
& \leq C_{1}\left[\int_{\Sigma_{1 T}}\left[z_{t}^{2}+v_{t}^{2}\right] d \Sigma_{1 T}+\int_{\Sigma_{1 T}}|\nabla z|^{2} d \Sigma_{1 T}\right. \\
& \left.\quad+E(\vec{z}, \vec{v}, T)+E(\vec{z}, \vec{v}, 0)+\int_{Q_{T}} z^{2} d Q_{T}\right]
\end{aligned}
$$

(where the constants $C_{0}$ and $C_{1}$ above are not necessarily the same throughout). Using (34) and the fact that on $\Gamma|\nabla z|^{2}=\left(\frac{\partial z}{\partial \nu}\right)^{2}+\left(\frac{\partial z}{\partial \tau}\right)^{2}$, we obtain the desired estimate (37), upon the addition of (44) and (45) and the use of the Neumann B.C.

Using estimates (33) and (37) in conjunction with the relation

$$
\begin{aligned}
\int_{0}^{T}\left\|A^{\frac{1}{2}} z\right\|_{L^{2}(\Omega)}^{2} d t & =\int_{Q_{T}} z_{t}^{2}-\left.\left(z_{t}, z\right)_{L^{2}(\Omega)}\right|_{0} ^{T} \\
& -\int_{0}^{T}\left(z_{t}, z\right)_{L^{2}\left(\Gamma_{1}\right)} d t+\int_{0}^{T}\left(v_{t}, z\right)_{L^{2}\left(\Gamma_{1}\right)} d t
\end{aligned}
$$

(obtained by multiplying (20) by $z$ and integrating from 0 to $T$ ), we then deduce the preliminary inequality

$$
\begin{aligned}
\int_{0}^{T} E(\vec{z}, \vec{v}, t) d t \\
\leq C\left[\int_{0}^{T}\left(\left\|\left.z_{t}\right|_{\Gamma_{1}}\right\|_{L^{2}\left(\Gamma_{1}\right)}^{2}+\left\|\AA^{\frac{1}{2}} v_{t}\right\|_{L^{2}\left(\Gamma_{1}\right)}^{2}\right) d t\right. \\
+\int_{\Sigma_{1 T}}\left(\frac{\partial z}{\partial \tau}\right)^{2} d \Sigma_{1 T}+E(\vec{z}, \vec{v}, T) \\
\left.+E(\vec{z}, \vec{v}, 0)+\|v\|_{L^{2}\left(\Sigma_{1 T}\right)}^{2}+\|z\|_{L^{2}\left(Q_{T}\right)}^{2}\right]
\end{aligned}
$$

Repeating the same argument as above, this time on the interval $(\epsilon, T-\epsilon)$, and further using the estimate (25) of Lemma A for the tangential derivative 
as well as the Neumann B.C. in (1), we arrive at

$$
\begin{aligned}
\int_{\epsilon}^{T-\epsilon} E(\vec{z}, \vec{v}, t) d t \\
\leq C_{T}\left[\int_{0}^{T}\left(\left\|\left.z_{t}\right|_{\Gamma_{1}}\right\|_{L^{2}\left(\Gamma_{1}\right)}^{2}+\left\|\AA \AA^{\frac{1}{2}} v_{t}\right\|_{L^{2}\left(\Gamma_{1}\right)}^{2}\right) d t\right. \\
\left.+\|v\|_{L^{2}\left(\Sigma_{1 T}\right)}^{2}+\|z\|_{H^{\frac{1}{2}+\epsilon}\left(Q_{T}\right)}^{2}\right] \\
\quad+C[E(\vec{z}, \vec{v}, T)+E(\vec{z}, \vec{v}, 0)]
\end{aligned}
$$

where the constant $C_{T}$ in (48) depends upon $T$, but $C$ does not. Using the relation (28) and its inherent dissipativity property, viz. $E(\vec{z}, \vec{v}, T) \leq$ $E(\vec{z}, \vec{v}, t) \forall 0 \geq t \geq T$, we have for $T>2 C+2 \epsilon$,

$$
\begin{aligned}
& E(\vec{z}, \vec{v}, T) \\
& \leq \frac{\left(C_{T}+2 C\right)}{(T-2 C-2 \epsilon)}\left[\int_{0}^{T}\left(\left\|\left.z_{t}\right|_{\Gamma_{1}}\right\|_{L^{2}\left(\Gamma_{1}\right)}^{2}+\left\|\AA^{\frac{1}{2}} v_{t}\right\|_{L^{2}\left(\Gamma_{1}\right)}^{2}\right) d t\right. \\
& \left.\quad+\|v\|_{L^{2}\left(\Sigma_{T}\right)}^{2}+\|z\|_{H^{\frac{1}{2}+\epsilon}\left(Q_{T}\right)}^{2}\right] .
\end{aligned}
$$

So with (49) in hand, the proof of Theorem 1 will be complete if we can "absorb" the lower order terms $\|v\|_{L^{2}\left(\Sigma_{1 T}\right)}^{2}$ and $\|z\|_{H^{\frac{1}{2}+\epsilon}\left(Q_{T}\right)}^{2}$, which we now proceed to do.

Lemma 3. Again, with the initial data $\left[\overrightarrow{z_{0}}, \overrightarrow{v_{0}}\right]$ in $D(\mathcal{A})$ and with $T$ sufficiently large, inequality (49) implies that there exists a positive constant $C_{T}$ such that

$$
\begin{aligned}
& \|v\|_{C\left([0, T] ; L^{2}\left(\Gamma_{1}\right)\right)}^{2}+\|z\|_{H^{\frac{1}{2}+\epsilon}\left(Q_{T}\right)}^{2} \\
& \leq C_{T}\left\{\int_{0}^{T}\left[\left\|\left.z_{t}\right|_{\Gamma_{1}}\right\|_{L^{2}\left(\Gamma_{1}\right)}^{2}+\left\|\AA^{\frac{1}{2}} v_{t}\right\|_{L^{2}\left(\Gamma_{1}\right)}^{2}\right] d t\right\} .
\end{aligned}
$$

Proof. We make use here of a compactness/uniqueness argument. If the lemma is false, then there exists a sequence $\left\{\overrightarrow{z_{0}^{(n)}}, \overrightarrow{v_{0}^{(n)}}\right\}_{n=1}^{\infty} \subseteq D(\mathcal{A})$, and a corresponding solution sequence $\left\{\overrightarrow{z^{(n)}}, \overrightarrow{v^{(n)}}\right\}_{n=1}^{\infty}$ which satisfies

$$
\begin{aligned}
& \left\|v^{(n)}\right\|_{C\left([0, T] ; L^{2}\left(\Gamma_{1}\right)\right)}^{2}+\left\|z^{(n)}\right\|_{H^{\frac{1}{2}+\epsilon}\left(Q_{T}\right)}^{2}=1 \forall n, \\
& \int_{0}^{T}\left[\left\|\left.z_{t}^{(n)}\right|_{\Gamma_{1}}\right\|_{L^{2}\left(\Gamma_{1}\right)}^{2}+\left\|\AA^{\frac{1}{2}} v_{t}^{(n)}\right\|_{L^{2}\left(\Gamma_{1}\right)}^{2}\right] d t \rightarrow 0 \text { as } n \rightarrow \infty .
\end{aligned}
$$

(52) and (49) then implies that the sequence $\left\{E\left(\overrightarrow{z^{(n)}}, \overrightarrow{v^{(n)}}, T\right)\right\}_{n=1}^{\infty}$ is bound- 
ed (uniformly in $n$ ), and consequently, (28) will have that $\left\{E\left(\overrightarrow{z^{(n)}}, \overrightarrow{v^{(n)}}, 0\right)\right\}_{n=1}^{\infty}$ is bounded. There thus exists a subsequence, still denoted by $\left\{\overrightarrow{z_{0}^{(n)}}, \overrightarrow{v_{0}^{(n)}}\right\}_{n=1}^{\infty}$, and $\left[\overrightarrow{z_{0}}, \overrightarrow{v_{0}}\right] \in H_{1} \times H_{0}$ such that

$$
\begin{aligned}
& \overrightarrow{z_{0}^{(n)}} \rightarrow \overrightarrow{z_{0}} \text { in } H_{1} \text { weakly; } \\
& \overrightarrow{v_{0}^{(n)}} \rightarrow \overrightarrow{v_{0}} \text { in } H_{0} \text { weakly. }
\end{aligned}
$$

If we denote $\left[\widetilde{z}, \widetilde{z}_{t}, \widetilde{v}, \widetilde{v}_{t}\right] \equiv[\overrightarrow{\vec{z}}, \vec{v}]$ as the solution pair corresponding to the weak limits $\left[\overrightarrow{z_{0}}, \overrightarrow{v_{0}}\right]$, then a fortiori

$$
\left\{\overrightarrow{z^{(n)}}, \overrightarrow{v^{(n)}}\right\} \rightarrow[\overrightarrow{\widetilde{z}}, \overrightarrow{\widetilde{v}}] \text { in } L^{\infty}\left(0, T ; H_{1} \times H_{0}\right) \text { weak star. }
$$

Thus, $z^{(n)} \rightarrow \widetilde{z}$ weakly in $H^{1}\left(Q_{T}\right)$, and consequently by a classic compactness theorem (see [13], p. 99, Theorem 16.1),

$$
z^{(n)} \rightarrow \widetilde{z} \text { in } H^{\frac{1}{2}+\epsilon}\left(Q_{T}\right) \text { strongly. }
$$

Moreover, we deduce from (55) and a compactness result of Simon's (see [14], Corollary 4) that

$$
v^{(n)} \rightarrow \widetilde{v} \text { in } C\left([0, T] ; L^{2}\left(\Gamma_{1}\right)\right) \text { strongly. }
$$

Consequently, taking the limit in (51),

$$
\|\widetilde{v}\|_{C\left([0, T] ; L^{2}\left(\Gamma_{1}\right)\right)}^{2}+\|\widetilde{z}\|_{H^{\frac{1}{2}+\epsilon}\left(Q_{T}\right)}^{2}=1 \forall n .
$$

Furthermore, the continuity of the map defined in Proposition 1 and the convergence in (53)-(54) provide that

$$
\begin{aligned}
& \left.\left.z_{t}^{(n)}\right|_{\Gamma_{1}} \rightarrow \widetilde{z}_{t}\right|_{\Gamma_{1}} \text { weakly in } L^{2}\left(\Sigma_{1 T}\right) ; \\
& \AA^{\frac{1}{2}} v_{t}^{(n)} \rightarrow \AA^{\frac{1}{2}} \widetilde{v}_{t} \text { weakly in } L^{2}\left(\Sigma_{1 T}\right) ;
\end{aligned}
$$

this convergence above, considered with that in (52) and the uniqueness of weak limits, allows one to deduce that

$$
\begin{aligned}
\left.\widetilde{z}_{t}\right|_{\Gamma_{1}} & =0 ; \\
\widetilde{v}_{t} & =0 .
\end{aligned}
$$

From (62), $\widetilde{v}=$ constant, and combining this with the B.C. in (2) we have

$$
\widetilde{v}=0 .
$$


In dealing with the term $\widetilde{z}$, we bring forth the representation of the Hilbert space adjoint $\mathcal{A}^{*}$ given in [2] by

$$
\begin{aligned}
& \mathcal{A}^{*}=\left[\begin{array}{cccc}
0 & -\mathbf{I} & 0 & 0 \\
A & -A N N^{*} A & 0 & -A N \\
0 & 0 & 0 & -\mathbf{I} \\
0 & N^{*} A & \AA & -\AA
\end{array}\right], \\
& \text { with } D\left(\mathcal{A}^{*}\right)=\left\{\left[z_{1}, z_{2}, v_{1}, v_{2}\right] \in D\left(A^{\frac{1}{2}}\right) \times D\left(A^{\frac{1}{2}}\right) \times D\left(\AA^{\frac{1}{2}}\right) \times D\left(\AA^{\frac{1}{2}}\right)\right. \\
& \text { such that } \left.z_{1}-N N^{*} A z_{2}-N v_{2} \in D(A) \text { and } v_{1}-v_{2} \in D(\AA)\right\} .
\end{aligned}
$$

From $(62)-(63),[\overrightarrow{\widetilde{z}}, \overrightarrow{0}]$ is a weak solution of (1)-(2) and moreover $\left.\widetilde{z}_{t}\right|_{\Gamma_{1}}=$ 0 , so we deduce from Ball's Theorem (see [3]) and the structure of $\mathcal{A}^{*}$ the following equation which holds pointwise for all $[\vec{z}, \vec{v}] \in D\left(\mathcal{A}^{*}\right)$ :

$$
\begin{aligned}
& \frac{d}{d t}\left(\left[\begin{array}{c}
\overrightarrow{\vec{z}}(t) \\
\overrightarrow{0}
\end{array}\right],\left[\begin{array}{c}
\vec{z} \\
\vec{v}
\end{array}\right]\right)_{H_{1} \times H_{0}} \\
& \quad=-\left(A^{\frac{1}{2}} \widetilde{z}(t), A^{\frac{1}{2}} z_{2}\right)_{L^{2}(\Omega)}+\left(\widetilde{z}_{t}(t), A z_{1}\right)_{L^{2}(\Omega)}
\end{aligned}
$$

choosing in particular $[\vec{z}, \vec{v}] \equiv\left[N N^{*} A z, z, 0,0\right] \in D\left(\mathcal{A}^{*}\right)$, where $z \in D(A)$, we obtain from $(65)$ and $(61)$ that $\widetilde{z}_{t t}=-A \widetilde{z} \in\left[D\left(A^{\frac{1}{2}}\right)\right]^{\prime}$; so making the change of variable $p=\widetilde{z}_{t}$ we then have $\left.p\right|_{\Gamma_{1}}=0$, and furthermore, $p$ solves the following wave equation:

$$
\left\{\begin{array}{c}
p_{t t}=\Delta p \text { on } Q_{T} \\
\left.p\right|_{\Gamma}=0 \text { on } \Sigma_{T} \\
\left.\frac{\partial p}{\partial \nu}\right|_{\Gamma_{1}}=0 \text { on } \Sigma_{1 T} .
\end{array}\right.
$$

For $T$ sufficiently large, we will hence have by Holmgren's Uniqueness Theorem that $p=0$ which implies that $\widetilde{z}=0$, and this outcome coupled with (63) contradicts the equality (58), thereby proving the lemma.

With Lemma 3 in hand, the proof of Theorem 1 is now complete.

\section{REFERENCES}

[1] G. Avalos and I. Lasiecka, A differential Riccati equation for the active control of a problem in structural acoustics, IMA Preprint Series, \#1345, (to appear in JOTA).

[2] G. Avalos and I. Lasiecka, The strong stability of a semigroup arising from a coupled hyperbolic/parabolic system, IMA Preprint Series, \#1347, (to appear in Semigroup Forum).

[3] J. M. Ball, Strongly continuous semigroups, weak solutions, and the variation of constants formula, Proc. Amer. Math. Soc. 63 (1977) 370-373.

[4] H. T. Banks, W. Fang, R. J. Silcox and R. C. Smith, Approximation methods for control of acoustic/structure models with piezoceramic actuators, NASA Contract Report, \#189578.

[5] H. T. Banks and R. C. Smith, Feedback control of noise in a 2-D nonlinear structural acoustics model, Discrete Contin. Dynam. Systems, 1 (1995), 119-149. 
[6] C. Bardos, G. Lebeau and J. Rauch, Control and stabilisation de l'equation des ondes, Appendix II in Contrôllabilité Exacte des Systèmes Distribués (by J.-L. Lions), Collection RMA, 8, Masson, Paris, 1988.

[7] G. Chen, A note on the boundary stabilization of the wave equation, SIAM J. Control Optim. 19 (1981), 106-113.

[8] S. Chen and R Triggiani, Proof of extensions of two conjectures on structural damping for elastic systems, Pacific J. Math. 136 (1989), 15-55.

[9] A. Bensoussan, G. Da Prato, M. Delfour and S. Mitter, Representation and Control of Infinite Dimensional Systems, I, Birkhäuser, Boston, 1993.

[10] P. Grisvard, Caracterization de qualques espaces d'interpolation, Arch. Rational Mech. and Anal. 25 (1967), 40-63.

[11] J. Lagnese, Decay of solutions of wave equations in a bounded region with boundary dissipation, J. Differential Equations 50 (1983), 163-182.

[12] I. Lasiecka and R. Triggiani, Uniform stabilization of the wave equation with Dirichlet or Neumann feedback control without geometrical conditions, Appl. Math. Optim. 25 (1992), 189-224.

[13] J. L. Lions and E. Magenes, Non-Homogeneous Boundary Value Problems and Applications, Springer-Verlag, New York, 1972.

[14] J. Simon, Compact sets in the space $L^{p}(0, T ; B)$, Ann. Mat. Pura Appl. (4) 148 (1987), $65-96$.

[15] R. Triggiani, Wave equation on a bounded domain with boundary dissipation: an operator approach, J. Math. Anal. Appl. 137 (1989), 438-461.

Institute for Mathematics and its Applications, University of Minnesota, MinNEAPOLIS, MN 55455-0436

E-mail address: avalos@ima.umn.edu 


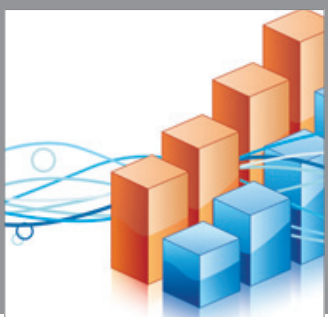

Advances in

Operations Research

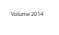

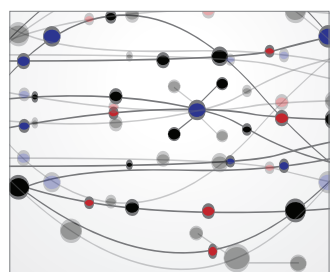

\section{The Scientific} World Journal
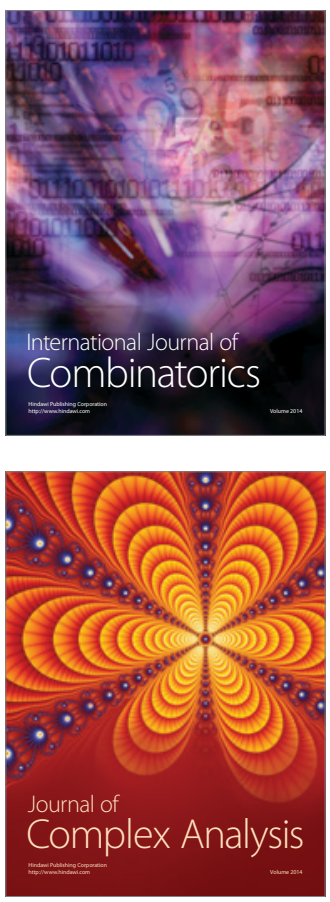

International Journal of

Mathematics and

Mathematical

Sciences
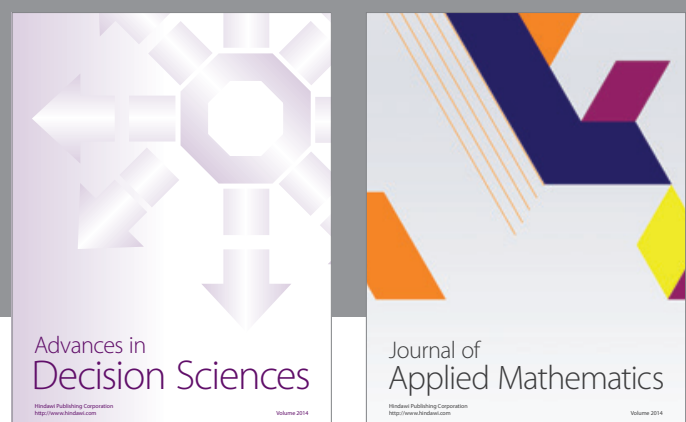

Journal of

Applied Mathematics
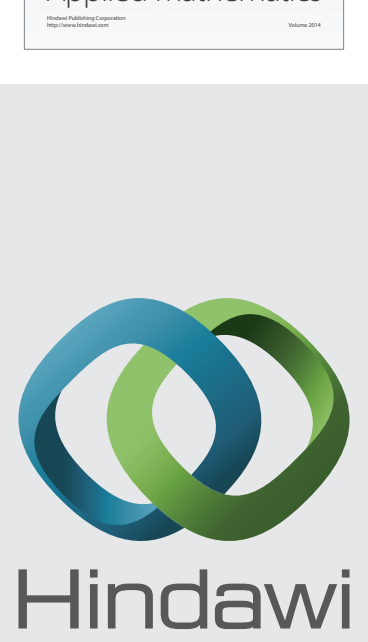

Submit your manuscripts at http://www.hindawi.com
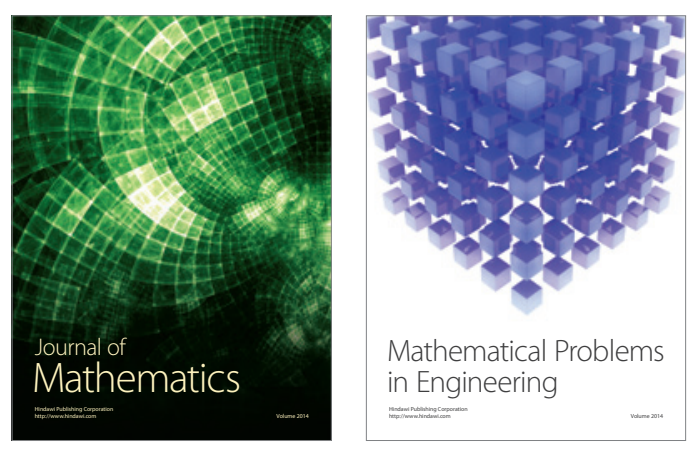

Mathematical Problems in Engineering
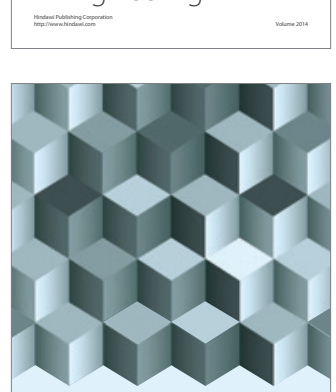

Journal of

Function Spaces
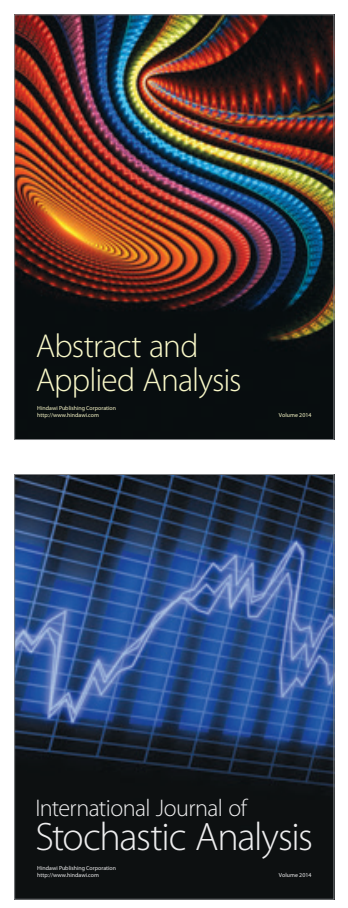

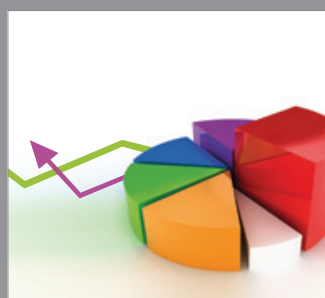

ournal of

Probability and Statistics

Promensencen
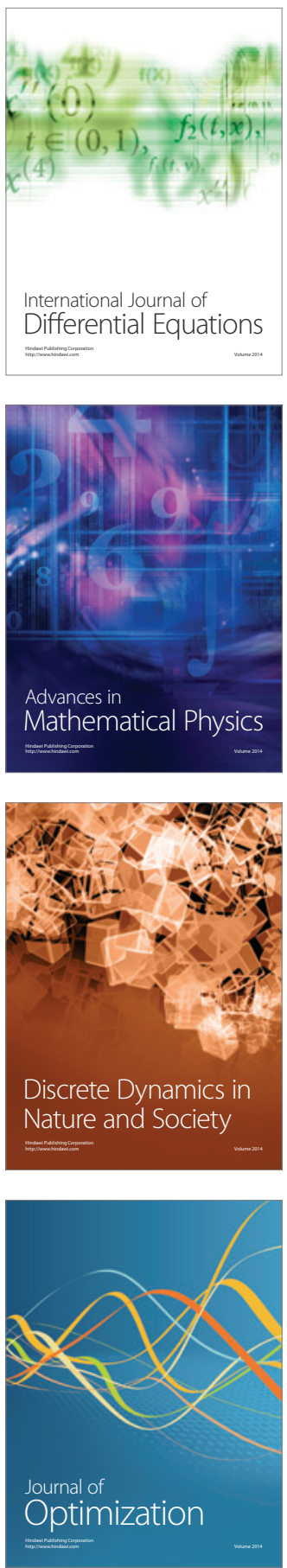